International Journal of Pure and Applied Mathematics

Volume 104 No. 2 2015, 171-180

ISSN: 1311-8080 (printed version); ISSN: 1314-3395 (on-line version)

url: http://www.ijpam.eu

doi: http://dx.doi.org/10.12732/ijpam.v104i2.2

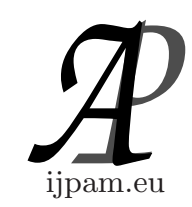

\title{
NUMERICAL SOLUTION OF NONLINEAR VOLTERRA-FREDHOLM INTEGRO DIFFERENTIAL EQUATION BY USING WALSH FUNCTIONS
}

\author{
R. Garshasbi ${ }^{1}$, A. Jafari Shaerlar ${ }^{2}$ \\ ${ }^{1,2}$ Department of Mathematics \\ Ardabil Science and research Branch \\ Islamic Azad University \\ Ardabil, IRAN \\ ${ }^{2}$ Department of Mathematics \\ Ardabil Branch \\ Islamic Azad University \\ Ardabil, IRAN
}

\begin{abstract}
In this article, we present a numerical method to solve the nonlinear Volterra-Fredholm integro-differential equations by new basis functions, in particular, Walsh functions. Then the operational matrix and direct method for solving the equations are investigated. Finally error estimates and numerical express are also reported.
\end{abstract}

AMS Subject Classification: 45G10, 45D05

Key Words: differential equations, direct method, Volterra-Fredholm integrodifferetial, error estimates, Walsh functions

\section{Introduction}

Several numerical methods for solving linear and nonlinear integro-differential equations have been presented. In most methods, a set of basis functions and an appropriate projection method such as Galerkin, collocation, eac. or a direct method have been applied, see [4].

Received: March 17, 2015

(c) 2015 Academic Publications, Ltd.

$\S$ Correspondence author url: www.acadpubl.eu 
In this work, we consider the Volterra-Fredholm integro-differential equations of the form

$$
\sum_{i=0}^{n} r_{i}(t) x^{(i)}(t)+\lambda_{1} \int_{0}^{s} k_{1}(s, t) F(x(t)) d t+\int_{0}^{1} k_{2}(x, t) G(x(t)) d t=y(t),
$$

where the functions $F(x(t))$ and $G(x(t))$ are polynomials of $x(t)$ with constant coefficients. For convenience, we put $F(x(t))=[x(t)]^{p}$ and $G(x(t))=[x(t)]^{q}$ where $\mathrm{p}$ and $\mathrm{q}$ are positive integers.

The presented method in this article can be extended and applied to solve Ordinary differential equations, nonlinear integral, and integro differential equa$\operatorname{tion}(1)$.

Many authors applied block pulse functions for solving differential problem, see $[1,6]$. For solving these equations, this paper uses the orthogonal Walsh functions and operational matrix for differential and integral equations.

\section{Block Pulse Functions (BPF)}

An m-set of BPF is defined as

$$
\phi(t)= \begin{cases}1 & (i-1) / m \leq t<i / m \\ 0 & \text { otherwise }\end{cases}
$$

the functions are disjoint and orthogonal. That is,

$$
\phi_{i}(t) \phi_{j}(t)=\left\{\begin{array}{ll}
\phi_{i}(t) & i=j \\
0 & i \neq j
\end{array}, \quad \phi_{i}(t) \phi_{j}(t)=\left\{\begin{array}{cc}
1 / m & i=j \\
0 & i \neq j
\end{array}\right.\right.
$$

The set $\left\{\phi_{i}(t)\right\}$ may be normalized to $\left\{\widehat{\phi_{i}(t)}\right\}$ by letting $\widehat{\phi_{i}(t)}=\sqrt{m} \phi_{i}(t)$ for all $i$. Thus $\left\{\widehat{\phi_{i}(t)}\right\}$ is a disjoint orthogonal system.

\subsection{Rademacher Functions (RF)}

RF's $\left\{r_{i}(t)\right\}$ on unit time are defined in the interval $[0,1)$. In general $r_{i}(t)$ is a train of unit pulses with $2^{m-1}$ cycles in $[0,1)$ taking alternately 1 and -1 . An exception is $r_{0}(t)$ which is the unit pulse over $[0,1)$. This system of square waves may be generated in many ways physically. The system $\left\{r_{i}(t)\right\}$ is orthonormal but not complete. 


\subsection{Walsh Functions (WF)}

WFs may be generated from RFs using the relation

$$
w_{n}(t)=\left[r_{q}(t)\right]^{d_{q}}\left[r_{q-1}(t)\right]^{d_{q-1}}\left[r_{q-1}(t)\right]^{d_{q-2}} \ldots
$$

where $w_{n}(t)$ is the $(n+1)$-th member of $w_{i}(t)$ ordered in a particular way, and $q=\left|\left[\log _{2}^{n}\right]\right|+1$ in which for natural number $m$ by $[m]$ least integer larger then over equal $m$. That is $n=d_{q} 2^{q-1}+d_{q-1} 2^{q-2}+\ldots$ and $d_{k}, k=0,1$ are binary digits of $n$. The system of WF's are orthonormal and complete.

\section{Relationships Among the Various Systems}

Consider the first $m=2^{k}$ ( $\mathrm{k}$ an integer) terms in each of the series of PCBF defined above and write them concisely as m-vectors

$$
\begin{aligned}
\phi(t) & =\left[\phi_{1}(t), \phi_{1}(t), \ldots, \phi_{1}(t)\right]^{T}, \quad r(t)=\left[r_{0}(t), r_{1}(t), \ldots, r_{m-1}(t)\right]^{T}, \\
w(t) & =\left[w_{0}(t), w_{1}(t), \ldots, w_{m-1}(t)\right]^{T} .
\end{aligned}
$$

All the PCBF may be expressed as linear combinations of BPF. As a result of such a possibility it can shown that

$$
W(t)=T_{B W} \phi(t), \quad r(t)=T_{B R} \phi(t)
$$

$T_{B W}$ is constant invertible matrixe. It is well known that the systems $\left\{w_{i}(t)\right\}$ is complete. With respect to the normalised system if we expand a function $f(t)$, the i-th Fourier coefficient is given by

$$
f_{i}=\frac{\left(f(t), \phi_{i}(t)\right)}{\left\|\phi_{i}(t)\right\|}=\sqrt{m} \int_{\frac{i-1}{m}}^{\frac{i}{m}} f(t) d t
$$

by virtue of the mean value theorem, we have $f_{i}^{2}=\frac{1}{m} f^{2}\left(t_{i}\right)$ where $t_{i} \in\left[\frac{i-1}{m}, \frac{i}{m}\right)$, $\lim _{m \rightarrow \infty} \sum_{i=1}^{m} f_{i}^{2}=\left\|f(t)^{2}\right\|$. The Fourier series with respect to $\left\{\phi_{i}(t)\right\}$ of $f(t)$ truncated to retain the first $m$ terms, may be compactly written as $f(t)=$ $\sum_{i=1}^{m} f_{i} \phi_{i}(t)=F^{T} \phi(t)$ where $F=\left[f_{1}, f_{2}, \ldots, f_{m}\right]^{T}$ for the particular cases $f(t)=$ $F_{W}^{T} W(t)$ where the vector $F_{W}$ represents the related spectra. 


\subsection{Integration with Respect to $t$}

Consider $\int_{0}^{t} \phi(t) d t$. Integrating each $\phi_{i}(t)$ in the integrand we get $\int_{0}^{t} \phi(t) d t=$ $E \phi(t)$ where

$$
E=\frac{1}{m}\left(\begin{array}{cccc}
\frac{1}{2} & 1 & \ldots & 1 \\
0 & \frac{1}{2} & \ldots & 1 \\
\vdots & \vdots & \ddots & \vdots \\
0 & 0 & \ldots & \frac{1}{2}
\end{array}\right)
$$

which may be concisely written as

$$
E=\frac{1}{m}\left[\frac{I}{2}+\sum_{i=1}^{m-1} \Delta^{i}\right]=\frac{1}{m}(I+\Delta)(I-\Delta)^{-1},
$$

if $f(t)=F^{T} \phi(t)$, an m-term approximation, the integral may be written as $\int_{0}^{t} F^{T} \phi(t) d t=F^{T} E \phi(t)$ where $E$ is defined as above constant invertible matrix.

\subsection{Differentiation}

In view of the fact that $\int_{0}^{t} f^{\prime}(s) d s=f(t)-f(0)$ by virtue of the approximation discussed, we get $f_{d}=E^{-1}(F-f(0) U)$ where $f_{d}$ and $U$ represent the PCBF spectral vectors of $f(t)$ and unit step function $[1,0,0, \ldots, 0]$ respectively. and by applying for hight-order we have

$$
\begin{aligned}
& \frac{d^{n} f(t)}{d t^{n}} \\
& =\left[\left(E^{-1}\right)^{n} F-\left(E^{-1}\right)^{n-1} f(0) U-\left(E^{-1}\right)^{n-2} f^{\prime}(0) U-\ldots-f^{(n-1)}(0) U\right]^{T} \phi(t),
\end{aligned}
$$

where

$$
E^{-1}=2 m(I-\Delta)(I+\Delta)^{-1}=2 m\left(I-2 \Delta+2 \Delta^{2}+\ldots\right) .
$$

\section{Error Estimated}

i) Error in PCBF approximation:

$$
e(t)=\sum_{i=1}^{m} f_{i} \phi(t)-f(t)
$$


ii) Error representation of the integral of PCBF:

$$
\mu_{i}(t)=\frac{f_{i}}{2 m}-f_{i}\left(t-\frac{i-1}{m}\right), \quad t \in\left[\frac{i-1}{m}, \frac{i}{m}\right) .
$$

iii)Error representation of the Differentiation of PCBF:

$$
\|\mu(t)\| \leq \frac{1}{2 \sqrt{3} m} \sup \left(f^{(n+1)}(\xi), \quad \xi \in[0,1) .\right.
$$

\section{Vector Forms}

Consider the $m$ terms of BPFs and write them concisely as m-vector: $\phi(t)=$ $\left[\phi_{0}(t), \phi_{1}(t), \ldots, \phi_{m-1}(t)\right]^{T}$. Above representation and disjointness property, follows:

$$
\begin{aligned}
\phi(t) \phi^{T}(t) & =\left(\begin{array}{cccc}
\phi_{0}(t) & 0 & \ldots & 0 \\
0 & \phi_{1}(t) & \ldots & 0 \\
\vdots & \vdots & \ddots & \vdots \\
0 & 0 & \ldots & \phi_{m-1}(t)
\end{array}\right), \\
\int_{0}^{1} \phi(t) \phi^{T}(t) d t & =\left(\begin{array}{cccc}
h & 0 & \ldots & 0 \\
0 & h & \ldots & 0 \\
\vdots & \vdots & \ddots & \vdots \\
0 & 0 & \ldots & h
\end{array}\right)=h I_{m \times m}=D, \\
\phi^{T}(t) \phi(t) & =1, \quad \phi(t) \phi^{T}(t) V=\widetilde{V} \phi(t),
\end{aligned}
$$

where, $V$ is an m-vector and $\widetilde{V}=\operatorname{diag}(V)$. Moreover, it can be clearly concluded that for every $m \times m$ matrix $B$ :

$$
\phi^{T}(t) B \phi(t)=\widehat{B^{T}} \phi(t),
$$

where, $\widehat{B}$ is an $\mathrm{m}=$ vector with elements equal to the diagonal entries of matrix $B$.

So for Walsh functions we have:

$$
\begin{gathered}
w^{T}(t) w(t)=\phi T(t) T_{B W}^{T} T_{B W} \phi(t)=T_{B W}^{T T_{B W}} \phi(t) \\
\int_{0}^{1} w(t) w^{T}(t) d t=\int_{0}^{1} T_{B W} \phi(t) \phi^{T}(t) T_{B W}^{T} d t \\
=T_{B W}\left(\int_{0}^{1} \phi(t) \phi^{T}(t) d t\right) T_{B W}^{T}=T_{B W} D T_{B W}^{T}
\end{gathered}
$$




\section{Method of Solution}

We consider special cases of Volterra-Fredholm integro-differential equations of the following form:

$$
x^{\prime}(t)+r(t) x(t)+\lambda_{1} \int_{0}^{t} k_{1}(s, t)(x(s))^{p} d s+\int_{0}^{1} k_{2}(s, t)(x(s))^{q} d s=y(t) .
$$

In this section, we solve Volterra-Fredholm integro-differential equations by using Walsh functions.

We now approximate functions $x(t), x^{\prime}(t),[x(t)]^{p},[x(t)]^{q}, k_{1}(s, t)$ and $k_{2}(s, t)$ with respect to walsh functions.

$$
\begin{aligned}
x(t) & =X^{T} w(t)=w^{T}(t) X, & y(t) & =Y^{T} w(t)=w^{T}(t) Y, \\
r(t) & =R^{T} w(t)=w^{T}(t) R, & x^{\prime}(t) & =\left[E^{-1}(X \ldots x(0) U)\right]^{T} w(t), \\
x^{p}(t) & =w(t)^{T} \Lambda=\Lambda^{T} w(t), & x^{q}(t) & =w(t)^{T} \Theta=\Theta^{T} w(t), \\
k_{1}(s, t) & =w^{T}(s) K_{1} w(t), & k_{2}(s, t) & =w^{T}(s) K_{2} w(t) .
\end{aligned}
$$

where $w(T)$ is defined above, the vectors $X, Y, R$ are walsh functions coefficients of $x(t), y(t), r(t)$, and, are column vectors whose elements are p-th and q-th powers of the vector $X$ and matrixes $K_{1}$ and $K_{2}$ are $m \times m$ walsh coefficients of $k_{1}(s, t)$ and $k_{2}(s, t)$ respectively.

To approximate the integrals, we get

$$
\begin{aligned}
\int_{0}^{t} k_{1}(s, t)[x(t)]^{p} d s \simeq \int_{0}^{s} w^{T}(t) K_{1} w(t) w^{T}(s) \Lambda d s & \\
= & {\left[T_{B W}^{T} K_{1} \widetilde{T_{B W}\left(\widetilde{T_{B W} \Lambda}\right) E}\right] \phi(t), }
\end{aligned}
$$

and so

$$
\begin{aligned}
\int_{0}^{1} k_{2}(s, t)[x(s)]^{q} d s & \simeq \int_{0}^{1} w^{T}(t) K_{2} w(s) w^{T}(s) \Theta d s \\
& =\phi^{T}(t) T_{B W}^{T} K_{2} T_{B W} \int_{0}^{1} \phi(s) \phi^{T}(s) T_{B W}^{T} \Theta d s \\
& =\phi^{T}(t) T_{B W}^{T} K_{2} T_{B W} D T_{B W}^{T} \Theta \\
& =\left[T_{B W}^{T} K_{2} T_{B W} D T_{B W}^{T} \Theta\right]^{T} \phi(t)
\end{aligned}
$$

by substituting above equations in Volterra-Fredholm integro-differential equations give 


$$
\begin{aligned}
& {\left[E^{-1}(X-x(0) U)\right]^{T} T_{B W} \phi(t)} \\
& +\left(T_{B W}^{T} \widehat{\widehat{Q X}^{T}} T_{B W}\right) \phi(t)+\lambda_{1}\left[T_{B W}^{T} K_{1} \widehat{\left.T_{B W}\left(\widetilde{T_{B W} \Lambda}\right) E\right] \phi(t)}\right. \\
& \quad+\lambda_{2}\left[T_{B W}^{T} K_{2} T_{B W} D T_{B W}^{T} \Theta\right]^{T} \phi(t)=Y_{T} T_{B W} \phi(t),
\end{aligned}
$$

or

$$
\begin{aligned}
{\left[E^{-1}(X-x(0) U)\right]^{T} T_{B W}+\left(T_{B W}^{T} \widehat{\widehat{Q X^{T}}} T_{B W}\right)+\lambda_{1}\left[T_{B W}^{T} K_{1} \widehat{\left.T_{B W}\left(\widetilde{T_{B W} \Lambda}\right) E\right]}\right.} \\
+\lambda_{2}\left[T_{B W}^{T} K_{2} T_{B W} D T_{B W}^{T} \Theta\right]^{T}=Y^{T} T_{B W}
\end{aligned}
$$

where given equation is a nonlinear system of $m$ algebraic equations for the $m$ unknowns $x_{0}, x_{1}, \ldots, x_{m-1}$ components of $X$ can be obtained by an iterative method. Hence, an approximate $x(t) \simeq X^{T} \Phi(t)$ can be computed for VolterraFredholm integro-differential equation without any projection method.

\subsection{Error Estimated for Method}

Now we consider the following erro estimates:

$$
\begin{aligned}
& x^{\prime}(t) \simeq x_{m}^{\prime}(t), \quad r(t) \simeq r_{m}(t), \quad y(t) \simeq y_{m}(t), \\
& x^{p}(t) \simeq x_{m}^{p}(t), \quad x^{q}(t) \simeq x_{m}^{q}(t) .
\end{aligned}
$$

By applying this approximations in the equation (1), we get

$$
\begin{aligned}
x_{m}^{\prime}(t)+r_{m}(t) x_{m}(t)+\lambda_{1} \int_{0}^{t}\left(k_{1}\right)_{m}(s, t) & \left(x_{m}(s)\right)^{p} d s \\
& +\int_{0}^{1}\left(k_{2}\right)_{m}(s, t)\left(x_{m}(s)\right)^{q} d s=y_{m}(t) .
\end{aligned}
$$

so by applying errors in (2), (3) and (4) we have

$$
\begin{aligned}
\|e r(t)\| \leq & \frac{1}{2 \sqrt{3} m} \sup \left(x^{\prime \prime}(\xi)+\|r(t)\| \frac{1}{2 \sqrt{3} m} \sup \left(x^{\prime}(\xi)\right.\right. \\
& +\|x(t)\| \frac{1}{2 \sqrt{3} m} \sup \left(r^{\prime}(\xi)\right) \\
& +\left|\lambda_{1}\right|\left\|k_{1}(\xi, t)\right\| \frac{1}{2 \sqrt{3} m} \sup \left(\left(x^{p}\right)^{\prime}(\xi)\right)+\left\|x^{p}(t)\right\| \frac{1}{2 \sqrt{3} m} \sup \left(k_{1}(t, \xi)\right) \\
& +\left\|k_{2}(\xi, t)\right\| \frac{1}{2 \sqrt{3} m} \sup \left(x^{q}(\xi)\right)+\left\|x^{q}(t)\right\| \frac{1}{2 \sqrt{3} m} \sup \left(k_{2}(t, \xi)\right) \\
& +\frac{1}{2 \sqrt{3} m} \sup \left(y^{\prime}(\xi) .\right.
\end{aligned}
$$




\begin{tabular}{||c|c|c|c||}
\hline \hline $\mathrm{t}$ & Exact solution & Approximate solution $\mathrm{m}=16$ & Approximate solution $\mathrm{m}=32$ \\
\hline 0.1 & 1.105171 & 1.106423 & 1.00086 \\
0.2 & 1.221403 & 1.216341 & 1.00050 \\
0.3 & 1.349859 & 1.354734 & 1.04311 \\
0.4 & 1.491825 & 1.494532 & 1.20501 \\
0.5 & 1.648721 & 1.647856 & 1.35822 \\
0.6 & 1.822119 & 1.821298 & 1.40134 \\
0.7 & 2.013753 & 2.001784 & 1.89030 \\
0.8 & 2.225541 & 2.215427 & 1.89883 \\
0.9 & 2.459603 & 2.452318 & 2.00003 \\
\hline \hline
\end{tabular}

Table 1
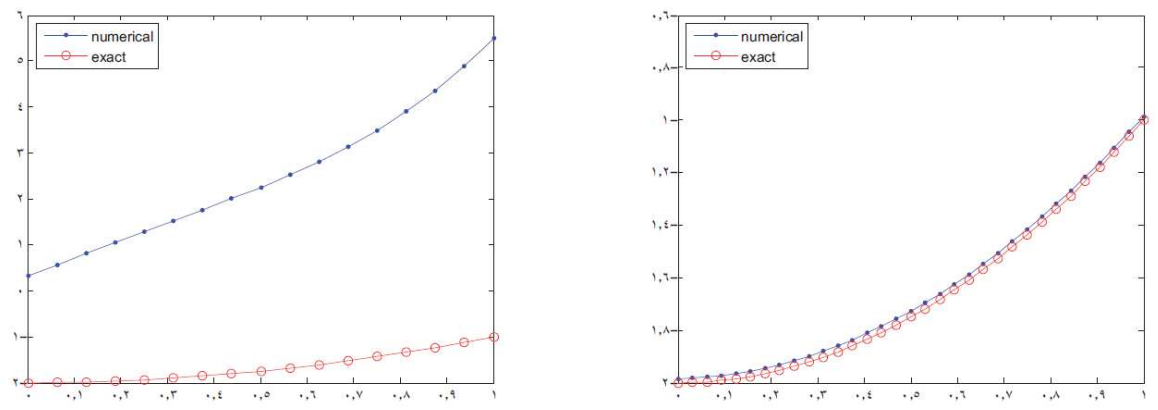

Figure 1: Exact solution and approximate solution for $m=16$ and $m=32$, respectively

\section{Example}

We consider the nonlinear Volterra-Fredholm integro-differential equation

$$
x^{\prime}(t)+2 t x(t)-\int_{0}^{t}(s+t) x^{3}(s) d s-\int_{0}^{1}(t-s) x(s) d s=y(t),
$$

where

$$
y(t)=\left(-\frac{2}{3} t+\frac{1}{9}\right) e^{3 t}+(2 t+1) e^{t}+\left(\frac{4}{3}-e^{1}\right) t+\frac{8}{9},
$$

with the initial condition $x(0)=1$, and the exact solution $x(t)=e^{t}$. Table 1 shows the numerical results. 


\section{Conclusion}

The approach presented transforms a nonlinear Volterra-Fredholm integro differential equation into a system of nonlinear algebric equations. The numerical results show that the accuracy of the solutions obtained is good. The approximate solutions by MATLAB software show the validity and efficiency of the proposed method.The above example shows that error will decrease as $m$ increases.This method can be easily extended and applied to system of nonlinear Volterra-Fredholm integro differential equation.

\section{Acknowledgments}

The authors would like to thank the refree for helpful suggestions.

\section{References}

[1] A.A. Dascioglu, A Chebyshev polynomial approch for linear FredholmVolterra integro-differential equations in the most general form, Appl. Math. Comput., 181 (2006), 103-112.

[2] A.V. Balakerishnan, M. Thoma, Control and Information Scinces, Springer-verlag. PB:419 (1983).

[3] A.M. Wazwaz, A reliable treatment for mixed Volterra-Fredholm integral equations, Appl. Math Comput. (2002), 127-405.

[4] E. Babolian, A. Salimi Shamloo, Numerical solution of Volterra integral and integro-differential equations of convolution type by using operational matrix of piecewise constant orthogonal functions, Journal of Comptational and Applied Math., 214 (2008), 495-508.

[5] E. Babolian, Z. Masouri, S. Hatamzadeh-Varmazyar, New direct method to solve nonlinear Volterra-Fredholm integral and integro-diffrential equations using operational matrix with Block-Pulse functions, Electromegnetics Research B, 8 (2008), 59-76.

[6] H. Brunner, On the numerical solution of nonlinear Volterra-Fredholm integral equation by collocation methods, SIAM J. Numer. Anal., 27, No. 4 (1990), 87. 
[7] K. Atkinson, The numerical solution of integral equations of the second kind, SIAM. J, No. 12 (1967).

[8] K. Maleknejad, M. Hadizadeh, The numerical analysis of Adomian's decomposition method for nonlinear Volterra integral and integro-differential equations, International J. Engineering Science, Iran University of Science and Technology, 8, No. 2a (1997), 33-48.

[9] M. Thoma, W. Wyner, Lecture Note in Contorol and Informationsciences, Springer-Vrrlag, ISBN: 3-540 55 369-X. 